\title{
cAMP regulates ADP-induced HSP27 phosphorylation in human platelets
}

\author{
YUKIKO ENOMOTO $^{1}$, SEIJI ADACHI ${ }^{2}$, TOMOAKI DOI ${ }^{3}$, HIDEO NATSUME $^{2,4}$, \\ KENJI KATO $^{2,4}$, RIE MATSUSHIMA-NISHIWAKI ${ }^{2}$, SHIGERU AKAMATSU ${ }^{5}$, \\ HARUHIKO TOKUDA ${ }^{6}$, SHINICHI YOSHIMURA ${ }^{1}$, TAKANOBU OTSUKA ${ }^{4}$, \\ SHINJI OGURA ${ }^{3}$, OSAMU KOZAWA ${ }^{2}$ and TORU IWAMA ${ }^{1}$
}

\begin{abstract}
Departments of ${ }^{1}$ Neurosurgery, ${ }^{2}$ Pharmacology, ${ }^{3}$ Emergency and Disaster Medicine, Gifu University Graduate School of Medicine, Gifu; ${ }^{4}$ Department of Orthopedic Surgery, Nagoya City University Graduate School of Medical Sciences, Nagoya; ${ }^{5}$ Department of Intensive Care Medicine, Matsunami General Hospital, Gifu;

${ }^{6}$ Department of Clinical Laboratory, National Center for Geriatrics and Gerontology, Obu, Japan
\end{abstract}

Received December 16, 2010; Accepted January 28, 2011

DOI: $10.3892 / \mathrm{ijmm} .2011 .637$

\begin{abstract}
Elevation of cAMP in platelets is recognized to play a suppressive role in platelet functions. We have previously shown that adenosine diphosphate (ADP)-induced phosphorylation of heat shock protein 27 (HSP27) via p38 mitogen-activated protein (MAP) kinase is correlated with platelet-derived growth factor (PDGF)-AB secretion and soluble CD40 ligand (sCD40L) release. In the present study, we investigated the relationship between cAMP and HSP27 phosphorylation in platelet function. 8-Bromoadenosine3',5'-cyclic monophosphate (8-bromo-cAMP), a plasma membrane-permeable cAMP analogue, or cilostazol, an inhibitor of cAMP phosphodiesterase, markedly attenuated the ADP-induced phosphorylation levels of p38 MAP kinase. In addition, the ADP-induced HSP27 phosphorylation was suppressed by 8-bromo-cAMP or cilostazol. 8-Bromo-cAMP, forskolin and cilostazol remarkably reduced the ADP-stimulated PDGF-AB secretion and SCD40L release. These results strongly suggest that cAMP regulates ADP-stimulated platelet activation due to inhibition of HSP27 phosphorylation via p38 MAP kinase.
\end{abstract}

\section{Introduction}

Platelet adhesion and aggregation represents the first step in thrombogenesis. Platelets are activated by a variety of stimuli, resulting in shape change, adhesion, aggregation, and

Correspondence to: Dr Osamu Kozawa, Department of Pharmacology, Gifu University Graduate School of Medicine, Yanagido1-1, Gifu 501-1194, Japan

E-mail: okozawa@gifu-u.ac.jp

Key words: cAMP, cilostazol, adenosine diphosphate, DP, heat shock protein 27 , platelet, phosphorylation subsequently thrombus formation. Platelet aggregation plays a pivotal role in thrombus formation. Thrombus formation provokes platelet granule secretion, such as platelet-derived growth factor (PDGF)-AB and serotonin (5-HT), and release of inflammatory substances, such as soluble CD40 ligand (sCD40L) (1).

Adenosine diphosphate (ADP) activates human platelets through P2-receptors such as P2Y1, P2Y12 and P2X receptors $(2,3)$. Although ADP is considered to be a weak agonist compared to thrombin or collagen, ADP is an essential cofactor for the activation of platelets by other agonists $(4,5)$. Low concentrations of ADP amplify the agonist-induced platelet activation (6). It is well known that the activation of P2-receptors leads to both platelet aggregation through P2Y1 and $\mathrm{P} 2 \mathrm{Y} 12$ receptors and to shape changes through $\mathrm{P} 2 \mathrm{X}$ receptors (7). P2Y1 or P2Y12 receptor activation by ADP reportedly results in the activation of either p44/p42 mitogenactivated protein (MAP) kinase or p38 MAP kinase in human platelets (8).

In response to environmental stress such as heat and chemicals, heat shock proteins (HSPs) are expressed in a variety of cells (9). HSPs are classified into high-molecularweight HSPs and low-molecular-weight HSPs based on their apparent molecular weights. Low-molecular-weight HSPs with molecular masses from 10-30 kDa, such as HSP27, have high homology in their amino acid sequences (10). It is generally recognized that low-molecular-weight HSPs may have chaperoning functions similarly to the high-molecular-weight HSPs (10). It is recognized that HSP27 activity is regulated by post-translational modifications such as phosphorylation (11). HSP27 becomes rapidly phosphorylated in response to various stresses, as well as exposure to cytokines and mitogens $(12,13)$. Human HSP27 is phosphorylated at three serine residues (Ser-15, Ser-78 and Ser-82). While HSP27 exists in an aggregated form under unstimulated conditions, it rapidly dissociates as a result of stimulation-induced phosphorylation. It has been shown that HSP27 phosphorylation is catalyzed by members of the MAP kinase superfamily, such as the 
p38 MAP kinase, the stress-activated protein kinase/c-Jun N-terminal kinase (SAPK/JNK) and the p44/p42 MAP kinase $(14,15)$. ADP reportedly induces HSP27 phosphorylation in human platelets (16). We have recently shown that ADP-induced phosphorylation of HSP27 via p38 MAP kinase is correlated with PDGF-AB secretion and sCD40L release in human platelets $(17,18)$.

In the present study, we examined the relationship between cAMP and ADP-induced HSP27 phosphorylation in human platelets since it is recognized that cyclic nucleotide cAMP plays an inhibitory role in platelet function (19). We show that cAMP suppresses ADP-induced PDGF-AB secretion and SCD40L release due to inhibiting HSP27 phosphorylation via p38 MAP kinase in human platelets.

\section{Materials and methods}

Materials. 8-Bromoadenosine-3',5'-cyclic monophosphate (8-bromo-cAMP), ADP and forskolin were purchased from Sigma-Aldrich (St. Louis, MO). Cilostazol was kindly provided from Otsuka Pharmaceutical (Tokushima, Japan). p38 MAP kinase antibodies and phospho-p38 MAP kinase were from Cell Signaling, Inc. (Beverly, MA). HSP27 antibodies, phospho-HSP27 (Ser-15) antibodies and phospho-HSP27 (Ser-78) antibodies were from StressGen Biotechnologies (Victoria, BC, Canada). Phospho-HSP27 (Ser-82) was from Biomol Research Laboratories (Plymouth Meeting, PA). The GAPDH antibody was purchased from Santa Cruz Biotechnology, Inc. (Santa Cruz, CA). ECL Western blotting detection system was purchased from GE Healthcare (Buckinghamshire, UK). Other materials and chemicals were obtained from commercial sources.

Preparation of platelets. Human blood donated from healthy volunteers was stored in a $1 / 10$ volume of a $3.8 \%$ sodium citrate. Platelet-rich plasma (PRP) was obtained from blood samples by centrifugation at $150 \mathrm{x}$ g for $12 \mathrm{~min}$ at room temperature. Platelet-poor plasma was prepared from residual blood by centrifugation at $800 \mathrm{x}$ g for $5 \mathrm{~min}$. All participants signed an informed consent agreement after receiving a detailed explanation and the study was approved by the Committee of Ethics of the Gifu University Graduate School of Medicine.

Measurement of platelet aggregation induced by ADP. Platelet aggregation using citrated PRP was followed in a PA-200 aggregometer (Kowa Co. Ltd., Tokyo, Japan), which can determine the size of platelet aggregates based upon particle counting using laser scattering methods (small size, 9-25 $\mu \mathrm{m}$; medium size, 25-50 $\mu \mathrm{m}$; large size, 50-70 $\mu \mathrm{m})$ (20), at $37^{\circ} \mathrm{C}$ with a stirring speed of $800 \mathrm{rpm}$. The platelets were preincubated for $1 \mathrm{~min}$, and then platelet aggregation was monitored for $4 \mathrm{~min}$. The percentage of transmittance of the isolated platelets was recorded as $0 \%$, and that of the appropriate platelet-poor plasma (blank) was recorded as $100 \%$. When indicated, PRP was pretreated with 8-bromo-cAMP or cilostazol for $15 \mathrm{~min}$.

Protein preparation after ADP stimulation. After stimulation with ADP, platelet aggregation was terminated by the addition of an ice-cold EDTA $(10 \mathrm{mM})$ solution. The mixture was centrifuged at $10,000 \mathrm{x}$ g at $4^{\circ} \mathrm{C}$ for $2 \mathrm{~min}$. To measure PDGF-AB and sCD40L as described below, the supernatant was isolated and stored at $-20^{\circ} \mathrm{C}$ for subsequent enzymelinked immune-sorbent assay (ELISA). For Western blot analysis, the pellet was washed twice with phosphate-buffered saline and then lysed and immediately boiled in a lysis buffer containing $62.5 \mathrm{mM}$ Tris/ $\mathrm{HCl}, \mathrm{pH} 6.8 ; 2 \%$ sodium dodecyl sulfate (SDS); $50 \mathrm{mM}$ dithiothreitol; and 10\% glycerol.

Western blot analysis. Western blot analysis was performed as previously described (21). Briefly, SDS-PAGE was performed by the method of Laemmli (22) in a 12 or $10 \%$ polyacrylamide gel. Proteins were fractionated and transferred onto Immobillon-P membranes (PVDF). Membranes were blocked with $5 \%$ fat-free dry milk in Tris-buffered saline with $0.1 \%$ Tween-20 (TBS-T; 20 mM Tris, pH 7.6; $137 \mathrm{mM} \mathrm{NaCl;0.1 \%}$ Tween) for $2 \mathrm{~h}$ before incubation with primary antibodies against GAPDH, HSP27, phospho-HSP27 (Ser-15), phosphoHSP27 (Ser-78), phospho-HSP27 (Ser-82), p38 MAP kinase and phospho-p38 MAP kinase. Peroxidase-labeled antimouse IgG was used as a secondary antibody. The antibodies were diluted for optimum concentration with 5\% fat-free dry milk in TBS-T. Peroxidase activity on PVDF membranes was visualized on an X-ray film by means of an ECL Western blotting detection system.

Measurement of PDGF-AB and $s C D 40 L$ levels. The PDGF-AB and SCD40L levels in samples were determined using PDGF-AB Quantikine and sCD40-Ligand Quantikine ELISA purchased from R\&D Systems (Minneapolis, MN), respectively, according to the manufacturer's protocol.

Statistical analysis. The data were analyzed by ANOVA followed by the Bonferroni method for multiple comparisons between the indicated pairs or the paired t-test, and a $\mathrm{p}<0.05$ was considered significant. All data are presented as the mean \pm SEM.

\section{Results}

Effect of 8-bromo-cAMP and cilostazol on platelet aggregation stimulated by $A D P$. It is well known that cAMP elevation induces the suppression of platelet aggregation (19). We confirmed that 8-bromo-cAMP suppressed the ADP-induced platelet aggregation in a dose-dependent manner at concentrations from 0.3 and $3 \mu \mathrm{M}$. In addition, we showed that cilostazol, an inhibitor of cAMP phosphodiesterase (23), slightly reduced the ADP-stimulated platelet aggregation in a dose-dependent manner at concentrations ranging between 3 and $20 \mu \mathrm{M}$ (Fig. 1). According to the analysis of the size of the platelet aggregates, large aggregates $(50-70 \mu \mathrm{m})$ were dose-dependently decreased by cilostazole (Fig. 1). On the other hand, cilostazol increased small aggregates $(9-25 \mu \mathrm{m})$ in a dose-dependent manner (Fig. 1).

Effects of 8-bromo-cAMP or cilostazol on the ADP-induced phosphorylation of p38 MAP kinase or HSP27 in human platelets. We have previously demonstrated that ADP induces the phosphorylation of HSP27 via activation of p38 MAP 


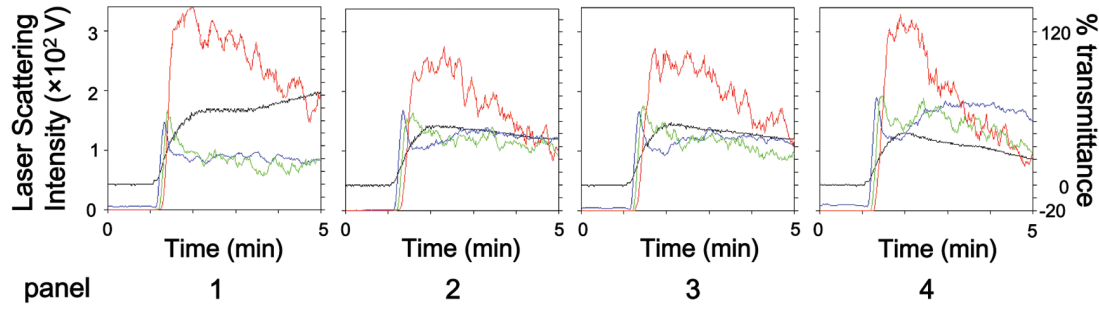

$\begin{array}{rcccc}\text { cilostazol }(\mu \mathrm{M}) & 0 & 3 & 10 & 20 \\ \text { ADP } & + & + & + & + \\ \text { ratio (\%) } & 22 & 30 & 29 & 34 \\ \text { small } & 20 & 28 & 27 & 29 \\ \text { medium } & 58 & 42 & 44 & 37 \\ \text { large } & 100 & 100 & 100 & 100\end{array}$

Figure 1. Effect of cilostazol on the ADP-induced platelet aggregation. Platelet-rich plasma (PRP) was pretreated with various doses of cilostazol at $37^{\circ} \mathrm{C}$ for $15 \mathrm{~min}$, and then stimulated by $3 \mu \mathrm{M}$ ADP for $4 \mathrm{~min}$. Finally, addition of ice-cold EDTA (10 mM) solution was used to terminate the reaction. The black line indicates the percentage of transmittance of each sample (the isolated platelets were recorded as 0\%, and platelet-free plasma was recorded as $100 \%$ ). The blue line indicates the small aggregates $(9-25 \mu \mathrm{m})$; the green line, the medium aggregates $(25-50 \mu \mathrm{m})$; the red line, the large aggregates $(50-70 \mu \mathrm{m})$. The distributions (\%) of aggregated particle size were measured with laser scattering methods.

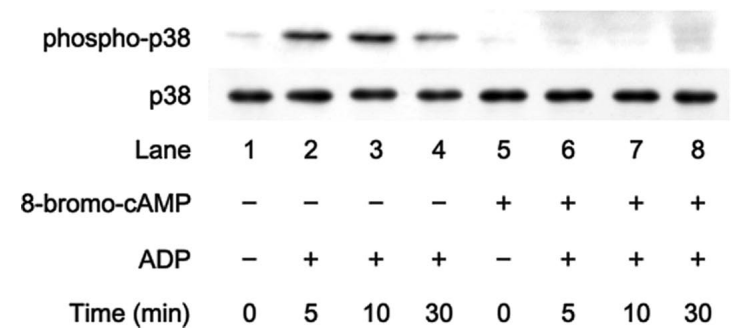

Figure 2. Effect of 8-bromo-cAMP on the ADP-induced phosphorylation of p38 MAP kinase in human platelets. Platelet-rich plasma (PRP) was pretreated with $1 \mathrm{mM} 8$-bromo-cAMP or vehicle at $37^{\circ} \mathrm{C}$ for $15 \mathrm{~min}$, and then stimulated by $3 \mu \mathrm{M}$ ADP for the indicated periods. The reaction was terminated by the addition of ice-cold EDTA $(10 \mathrm{mM})$ solution. The protein extracts were harvested as described in Materials and methods, and then subjected to SDS-PAGE using phospho-specific p38 MAP kinase and p38 MAP kinase antibodies. Representative results from at least three independent experiments are shown.

kinase in human platelets, resulting in secreting PDGF-AB and releasing $\mathrm{SCD} 40 \mathrm{~L}(17,18)$. We have previously demonstrated that 4 min-stimulation by ADP is sufficient for the activation of p38 MAP kinase (17). Therefore, we next examined the effects of 8-bromo-cAMP or cilostazole on the ADP-induced phosphorylation of p38 MAP kinase in human platelets. 8-Bromo-cAMP remarkably reduced the ADP-induced phosphorylation of p38 MAP kinase (Fig. 2). Similarly, cilostazol suppressed the ADP-induced phosphorylation of p38 MAP kinase dose-dependently in the concentration range of 3-20 $\mu \mathrm{M}$ (Fig. 3).

In our previous report (17), we showed that ADP stimulates HSP27 phosphorylation at three serine residues (Ser-15,

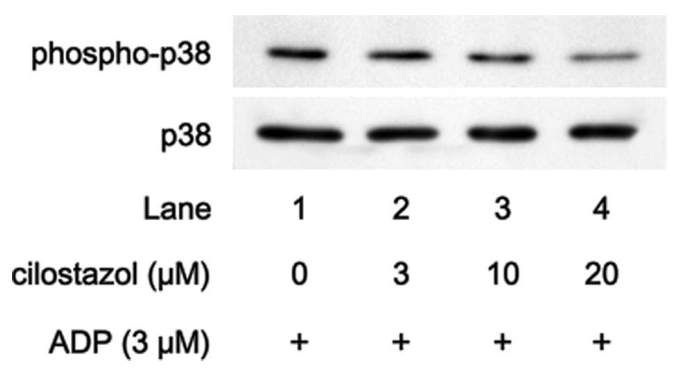

Figure 3. Effect of cilostazol on the ADP-induced phosphorylation of p38 MAP kinase in human platelets. Platelet-rich plasma (PRP) was pretreated with various doses of cilostazol at $37^{\circ} \mathrm{C}$ for $15 \mathrm{~min}$, and then stimulated by $3 \mu \mathrm{M}$ ADP for $5 \mathrm{~min}$. The reaction was terminated by the addition of ice-cold EDTA $(10 \mathrm{mM})$ solution. The protein extracts were harvested as described in Materials and methods, and then subjected to SDS-PAGE using phosphospecific p38 MAP kinase and p38 MAP kinase antibodies. Representative results from at least three independent experiments are shown.

Ser-78 and Ser-82) in human platelets. We further examined the effect of 8-bromo-cAMP on ADP-induced phosphorylation of HSP27 (Ser-15, Ser-78 and Ser-82). As shown in Fig. 4, the phosphorylation levels of HSP27 (Ser-15, Ser-78 and Ser-82) induced by ADP were almost completely suppressed by 8-bromo-cAMP. In addition, cilostazole attenuated the phosphorylated levels of HSP27 (Ser-15, Ser-78 and Ser-82) induced by ADP (Fig. 5) in a dose-dependent manner between 3 and $20 \mu \mathrm{M}$.

Effects of 8-bromo-cAMP or forskolin on the ADP-induced $P D G F-A B$ secretion and the $S C D 40 L$ release from human platelets. In our previous studies $(17,18)$, we have shown that 


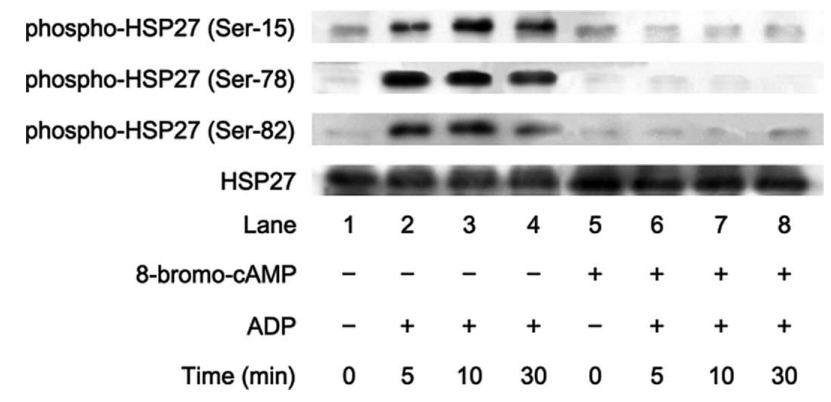

Figure 4. Effect of 8-bromo-cAMP on the phosphorylation of HSP27 in human platelets. Platelet-rich plasma (PRP) was pretreated with $1 \mathrm{mM}$ 8-bromo-cAMP or vehicle at $37^{\circ} \mathrm{C}$ for $15 \mathrm{~min}$, and then stimulated by $3 \mu \mathrm{M}$ ADP for $5 \mathrm{~min}$. The reaction was terminated by the addition of ice-cold EDTA $(10 \mathrm{mM})$ solution. The protein extracts were harvested as described in Materials and methods, and then subjected to SDS-PAGE using phosphospecific HSP27 (Ser-15, Ser-78 and Ser-82) and total HSP27 antibodies. Representative results from at least three independent experiments are shown.

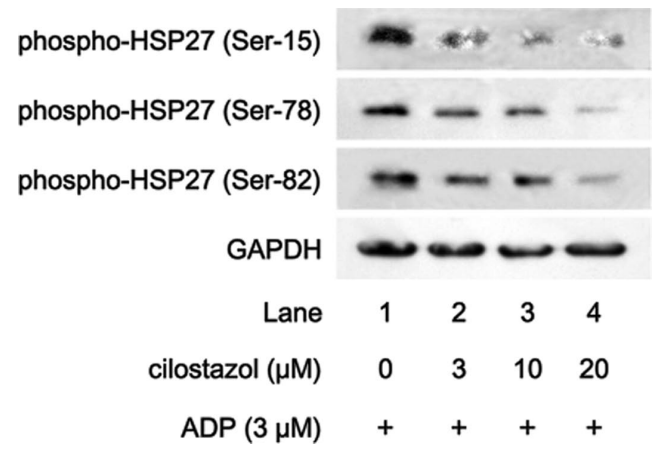

Figure 5. Effect of cilostazol on the phosphorylation of HSP27 in human platelets. Platelet-rich plasma (PRP) was pretreated with various doses of cilostazol at $37^{\circ} \mathrm{C}$ for $15 \mathrm{~min}$, and then stimulated by $3 \mu \mathrm{M}$ ADP for $5 \mathrm{~min}$. The reaction was terminated by the addition of ice-cold EDTA $(10 \mathrm{mM})$ solution. The protein extracts were harvested as described in Materials and Methods, and then subjected to SDS-PAGE using antibodies against phospho-specific HSP27 (Ser-15, Ser-78 and Ser-82) and GAPDH. Representative results from at least three independent experiments are shown.

ADP stimulates PDGF-AB granule secretion and sCD40L release at least in part via HSP27 phosphorylation through p38 MAP kinase activation from human platelets. We next examined the effect of 8-bromo-cAMP on the ADP-induced secretion of PDGF-AB and release of SCD40L from human platelets. 8-Bromo-cAMP significantly suppressed the ADP-induced PDGF-AB secretion and $\mathrm{SCD} 40 \mathrm{~L}$ release from human platelets (Fig. 6). Moreover, we examined the effect of forskolin, a direct activator of adenylyl cyclase (24), on the ADP-induced platelet responses. Forskolin dose-dependently inhibited the PDGF-AB secretion and $\mathrm{SCD} 40 \mathrm{~L}$ release at concentrations from 1-10 $\mu \mathrm{M}$ (Fig. 7).

Effect of cilostazol on the ADP-induced PDGF-AB secretion and $S C D 40 L$ release from human platelets. We next examined the effect of cilostazol on the ADP-induced secretion of PDGF-AB and release of SCD40L from human platelets. The ADP-stimulated secretion of PDGF-AB and release of SCD40L from human platelets were significantly reduced by cilostazol at concentrations ranging from 3-20 $\mu \mathrm{M}$ (Fig. 8).

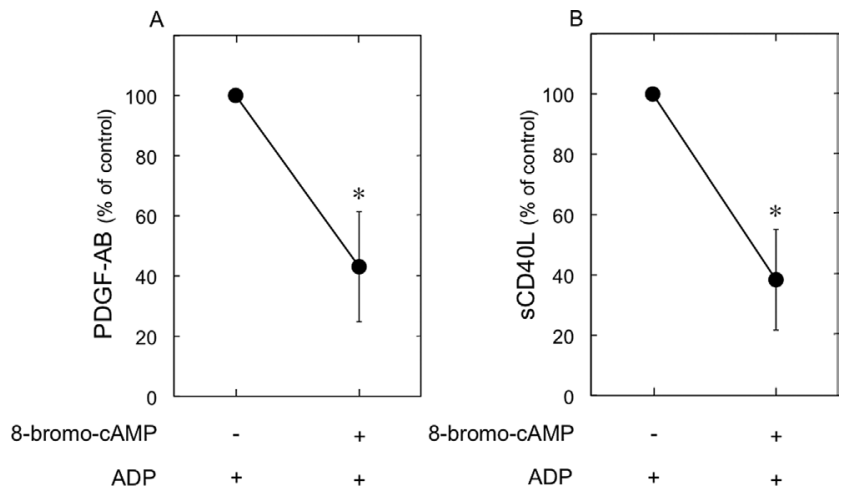

Figure 6. Effect of 8-bromo-cAMP on the ADP-induced platelet-derived growth factor (PDGF)-AB secretion (A) or $\mathrm{SCD} 40 \mathrm{~L}$ release (B) from human platelets. Platelet-rich plasma (PRP) was pretreated with $1 \mathrm{mM} 8$-bromocAMP or vehicle at $37^{\circ} \mathrm{C}$ for $15 \mathrm{~min}$, and then stimulated with $3 \mu \mathrm{M}$ ADP for $30 \mathrm{~min}$. The reaction was terminated by the addition of ice-cold EDTA $(10 \mathrm{mM})$ solution. The mixture was centrifuged at $10,000 \mathrm{x}$ g at $4^{\circ} \mathrm{C}$ and the supernatants were then subjected to the enzyme-linked immunosorbent assay for PDGF-AB or sCD40L. $n=6,{ }^{*} p<0.05$, compared to the value of ADP alone. Data were analyzed by the paired t-test. Each value represents the mean \pm SEM

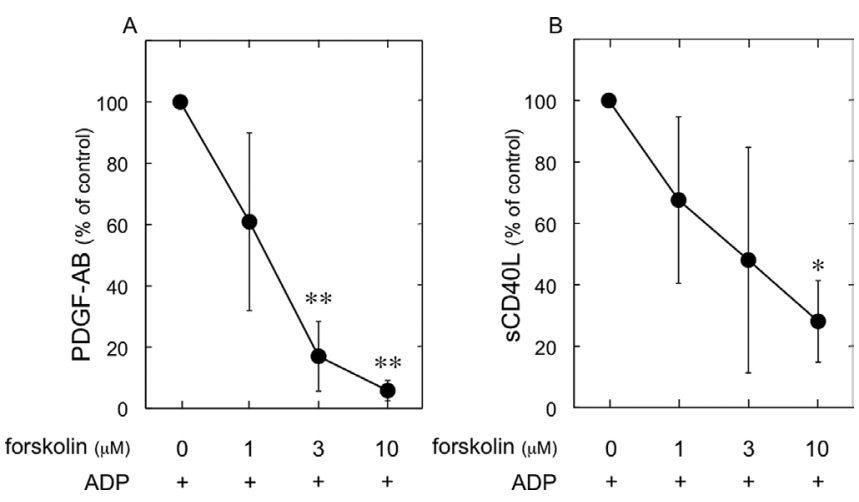

Figure 7. Effect of forskolin on the ADP-induced platelet-derived growth factor (PDGF)-AB secretion (A) or $\mathrm{SCD} 40 \mathrm{~L}$ release (B) from human platelets. Platelet-rich plasma (PRP) was pretreated with various doses of forskolin at $37^{\circ} \mathrm{C}$ for $15 \mathrm{~min}$, and then stimulated with $3 \mu \mathrm{M}$ ADP for $30 \mathrm{~min}$. The reaction was terminated by the addition of ice-cold EDTA $(10 \mathrm{mM})$ solution. The mixture was centrifuged at $10,000 \mathrm{x} \mathrm{g}$ at $4^{\circ} \mathrm{C}$ and the supernatants were then subjected to the enzyme-linked immunosorbent assay for PDGF-AB or $\mathrm{sCD} 40 \mathrm{~L} . \mathrm{n}=6,{ }^{*} \mathrm{p}<0.05,{ }^{*} \mathrm{p}<0.01$, compared to the value of ADP alone. Data were analyzed by the paired t-test. Each value represents the mean \pm SEM.

\section{Discussion}

In the present study, we focused on the relationship between cAMP and HSP27 phosphorylation in ADP-stimulated human platelets. First, we confirmed that 8-bromo-cAMP and cilostazol significantly suppressed ADP-induced platelet aggregation which is consistent with the previous study (19). We showed that cell membrane-permeable 8-bromo-AMP suppressed ADP-induced HSP27 phosphorylation (Ser-15, Ser-78 and Ser-82). In addition, we also demonstrated that the HSP27 phosphorylation at three serine residues by ADP was reduced by cilostazol, an inhibitor of cyclic nucleotide phosphodiesterase III that induces cAMP hydrolysis (23). Therefore, these findings suggest that cAMP elevation attenuates the ADP-induced HSP27 phosphorylation in human platelets. 


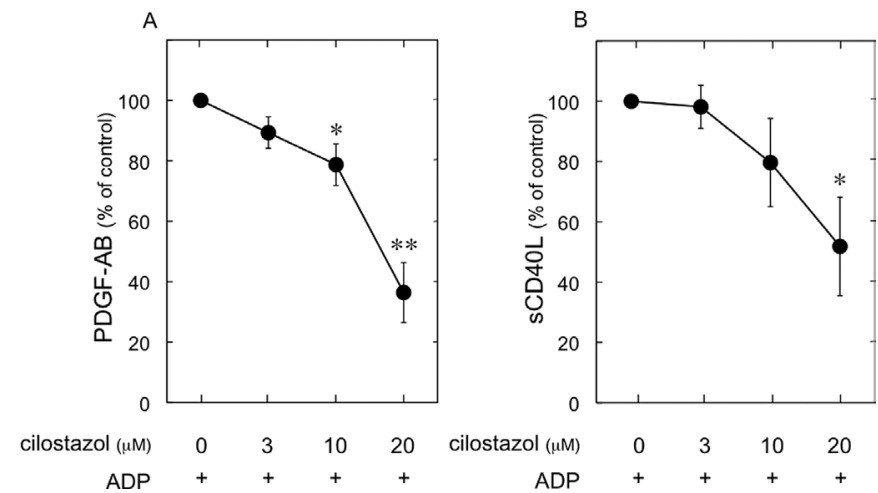

Figure 8. Effect of cilostazol on the ADP-induced platelet-derived growth factor (PDGF)-AB secretion (A) or $\mathrm{SCD} 40 \mathrm{~L}$ release (B) from human platelets. Platelet-rich plasma (PRP) was pretreated with various doses of cilostazol at $37^{\circ} \mathrm{C}$ for $15 \mathrm{~min}$, and then stimulated with $3 \mu \mathrm{M}$ ADP for $30 \mathrm{~min}$. The reaction was terminated by the addition of ice-cold EDTA $(10 \mathrm{mM})$ solution. The mixture was centrifuged at $10,000 \mathrm{x}$ at $4^{\circ} \mathrm{C}$ and the supernatants were then subjected to the enzyme-linked immunosorbent assay for PDGF-AB or sCD40L. $\mathrm{n}=6,{ }^{*} \mathrm{p}<0.05,{ }^{* *} \mathrm{p}<0.01$, compared to the value of ADP alone. Data were analyzed by the paired t-test. Each value represents the mean \pm SEM.

We have previously reported that ADP stimulates the HSP27 phosphorylation via p38 MAP kinase activation in human platelets (17). Thus, we next investigated the effect of cAMP on the ADP-induced activation of p38 MAP kinase. We showed that 8-bromo-cAMP and cilostazol remarkably reduced the p38 MAP kinase phosphorylation by ADP. MAP kinases are recognized to be activated by phosphorylation of threonine and tyrosine residues by the dual specificity MAP kinase kinase (25). Thus, it is likely that cytosolic cAMP elevation attenuates p38 MAP kinase activity under the stimulation of ADP. Taking our findings into account, it is most likely that the effect of cAMP is exerted at a point upstream from p38 MAP kinase in human platelets, resulting in the inhibition of HSP27 phosphorylation.

Among the $\mathrm{P} 2$ receptors, it is generally recognized that the $\mathrm{P} 2 \mathrm{Y} 12$ receptors play a central role in platelet activation when stimulated by $\operatorname{ADP}(2,3,26)$. Among the heterotrimeric GTP-binding protein family, the P2Y12 receptors couple primarily to the Gi protein which functions as an intermediary resulting in the inhibition of adenylyl cyclase. It has been reported that Akt, Rap and potassium channels are crucial functional effectors downstream of the P2Y12 receptor stimulation (26). On the other hand, the reduced levels of cAMP are not directly responsible for the downstream effects of the receptors (26). Interestingly, we herein showed that cilostazol, an inhibitor of cAMP phosphodiesterase, attenuated the phosphorylated levels of HSP27 in the ADP-stimulated platelets. Based on these findings, it is possible that the elevated cytosolic cAMP by cilostazol suppressed the secretion of PDGF-AB and the release of $\mathrm{SCD} 40 \mathrm{~L}$ due to the reduction of HSP27 phosphorylation in the P2Y12 receptor activation. Further investigation is necessary to clarify the exact mechanism of cAMP action in ADP-stimulated platelet functions.

It is well known that activated platelets secrete the materials stored in the specific granules such as densegranules and $\alpha$-granules. Dense-granules contain small non-protein molecules including ADP. On the other hand, $\alpha$-granules contain large adhesive and healing proteins such as PDGF-AB (1). PDGF-AB released from platelet $\alpha$-granules is a potent mitogenic growth factor, which mainly acts on connective tissue such as vascular smooth muscle cells and promotes arteriosclerosis (27). In addition, activated platelets release inflammatory mediators of atherosclerosis, such as CD40 ligand (CD40L). CD40L is stored in the cytoplasm of unstimulated platelets and rapidly translocates on the surface after platelet activation by agonists, such as $\operatorname{ADP}(28,29)$. The CD40L expressed on the activated platelet surface undergoes cleavage that generates a functional soluble fragment termed sCD40L. It is known that sCD40L release from platelets induces inflammatory responses via CD40, which is expressed on vascular endothelial cells and neurtrophils (30). We found that ADP-induced secretion of PDGF-AB from $\alpha$-granules was significantly inhibited by the pretreatment with 8-bromocAMP or cilostazol. In addition, the ADP-stimulated SCD40L release was also attenuated by 8-bromo-cAMP or cilostazol. Taking these findings and our results into account, it is most likely that elevation of cAMP suppresses the PDGF-AB secretion and the sCD40L release due to the inhibition of HSP27 phosphorylation via p38 MAP kinase in ADP-stimulated platelets. Cilostazol is recognized as an effective alternative to aspirin for prevention of stroke recurrence $(31,32)$. These findings could provide a novel mechanism of cilostazol as an anti-platelet medicine. In conclusion, our results strongly suggest that cAMP regulates ADP-stimulated platelet activation due to inhibiting HSP27 phosphorylation via p38 MAP kinase.

\section{Acknowledgements}

We are very grateful to Yoko Kawamura for her skillful technical assistance. This study was supported in part by a Grant-in-Aid for Scientific Research (20590565 and 20591825) from the Ministry of Education, Science, Sports and Culture of Japan and the Research Grants for Longevity Sciences (22A-4) and the Research on Proteomics from the Ministry of Health, Labour and Welfare of Japan.

\section{References}

1. Rendu F and Brohard-Bohn B: The platelet release reaction: granules' constituents, secretion and functions. Platelets 12: 261-273, 2001.

2. Hechler B, Leon C, Vial C, Vigne P, Frelin C, Cazenave JP and Gachet C: The P2Y1 receptor is necessary for adenosine 5'-diphosphate-induced platelet aggregation. Blood 92: 152-159, 1998.

3. Daniel JL, Dangelmaier C, Jin J, Kim YB and Kunapuli SP: Role of intracellular signaling events in ADP-induced platelet aggregation. Thromb Haemost 82: 1322-1326, 1999.

4. Colman RW: Aggregin: a platelet ADP receptor that mediates activation. FASEB J 4: 1425-1435, 1990.

5. Storey RF, Newby LJ and Heptinstall S: Effects of P2Y(1) and $\mathrm{P} 2 \mathrm{Y}(12)$ receptor antagonists on platelet aggregation induced by different agonists in human whole blood. Platelets 12: 443-447, 2001.

6. Kowalska MA, Ratajczak MZ, Majka M, Jin J, Kunapuli SP, Brass L and Poncz M: Stromal cell-derived factor-1 and macrophage-derived chemokine: 2 chemokines that activate platelets. Blood 96: 50-57, 2000.

7. Dangelmaier C, Jin J, Daniel JL, Smith JB and Kunapuli SP: The P2Y1 receptor mediates ADP-induced p38 kinase-activating factor generation in human platelets. Eur J Biochem 267: 2283-2289, 2000. 
8. Falker K, Lange D and Presek P: ADP secretion and subsequent P2Y12 receptor signalling play a crucial role in thrombininduced ERK2 activation in human platelets. Thromb Haemost 92: 114-123, 2004

9. Hendrick JP and Hartl FU: Molecular chaperone functions of heat-shock proteins. Annu Rev Biochem 62: 349-384, 1993.

10. Benjamin IJ and McMillan DR: Stress (heat shock) proteins: molecular chaperones in cardiovascular biology and disease. Circ Res 83: 117-132, 1998.

11. Welch WJ: Phorbol ester, calcium ionophore, or serum added to quiescent rat embryo fibroblast cells all result in the elevated phosphorylation of two 28,000-dalton mammalian stress proteins. J Biol Chem 260: 3058-3062, 1985.

12. Gaestel M, Schroder W, Benndorf R, Lippmann C, Buchner K, Hucho F, Frdmann VA and Bielka H: Identification of the phosphorylation sites of the murine small heat shock protein hsp25. J Biol Chem 266: 14721-14724, 1991.

13. Landry J, Lambert H, Zhou M, Lavoie JN, Hickey E, Weber LA and Anderson CW: Human HSP27 is phosphorylated at serines 78 and 82 by heat shock and mitogen-activated kinases that recognize the same amino acid motif as S6 kinase II. J Biol Chem 267: 794-803, 1992.

14. Kyriakis JM and Avruch J: Sounding the alarm: protein kinase cascades activated by stress and inflammation. J Biol Chem 271: 24313-24316, 1996.

15. Guay J, Lambert H, Gingras-Breton G, Lavoie JN, Huot J and Landry J: Regulation of actin filament dynamics by p38 map kinase-mediated phosphorylation of heat shock protein 27. J Cell Sci 110: 357-368, 1997

16. Zhu Y, O'Neill S, Saklatvala J, Tassi L and Mendelsohn ME: Phosphorylated HSP27 associates with the activation-dependent cytoskeleton in human platelets. Blood 84: 3715-3723, 1994.

17. Kato $H$, Takai S, Matsushima-Nishiwaki R, Adachi S, Minamitani C, Otsuka T, Tokuda H, Akamatsu S, Doi T, Ogura S and Kozawa O: HSP27 phosphorylation is correlated with ADP-induced platelet granule secretion. Arch Biochem Biophys 475: 80-86, 2008.

18. Doi T, Adachi S, Takai S, Matsushima-Nishiwaki R, Kato H, Enomoto Y, minamitani C, Otsuka T, Tokuda H, Akamatsu S, Iwama T, Kozawa $\mathrm{O}$ and Ogura S: Antithrombin III suppresses ADP-induced platelet granule secretion: inhibition of HSP27 phosphorylation. Arch Biochem Biophys 489: 62-67, 2009.

19. Schwarz UR, Walter U and Eigenthaler M: Taming platelets with cyclic nucleotides. Biochem Pharmacol 62: 1153-1161, 2001.

20. Fabre JE, Nguyen M, Latour A, Keifer JA, Audoly LP, Coffman TM and Koller BH: Decreased platelet aggregation, increased bleeding time and resistance to thromboembolism in P2Y1-deficient mice. Nat Med 5: 1199-1202, 1999.
21. Kato K, Ito H, Hasegawa K, Inaguma Y, Kozawa O and Asano T: Modulation of the stress-induced synthesis of hsp27 and alpha B-crystallin by cyclic AMP in C6 rat glioma cells. J Neurochem 66: 946-950, 1996

22. Laemmli UK: Cleavage of structural proteins during the assembly of the head of bacteriophage. Nature 227: 680-685, 1970.

23. O'Donnell MJ, Hankey GJ and Eikelboom JW: Antiplatelet therapy for secondary prevention of noncardioembolic ischemic stroke: a critical review. Stroke 39: 1638-1646, 2008.

24. Simonds WF: G protein regulation of adenylate cyclase. Trend Pharmacol Sci 20: 66-73, 1999.

25. Kyriakis JM and Avruch J: Mammalian mitogen-activated protein kinase signal transduction pathways activated by stress and inflammation. Physiol Rev 81: 807-869, 2001.

26. Dorsam RT, Kunapuli SP: Central role of the P2Y12 receptor in platelet activation. J Clin Invest 113: 340-345, 2004.

27. Heldin CH and Westermark B: Mechanism of action and in vivo role of platelet-derived growth factor. Physiol Rev 79: 1283-1316, 1999

28. Hermann A, Rauch BH, Braun M, Schror K and Weber AA Platelet CD40 ligand (CD40L): subcellular localization, regulation of expression, and inhibition by clopidogrel. Platelets 12: 74-82, 2001.

29. Andre P, Nannizzi-Alaimo L, Prasad SK and Phillips DR: Platelet-derived CD40L: the switch-hitting player of cardiovascular disease. Circulation 106: 896-899, 2002.

30. Henn V, Slupsky JR, Grafe M, Anagnostopoulos I, Forster R, Muller-Berghaus G and Kroczek RA: CD40 ligand on activated platelets triggers an inflammatory reaction of endothelial cells. Nature 391: 591-594, 1998.

31. Huang Y, Cheng Y, Wu J, Li Y, Xu E, Hong Z, Li Z, Zhang W, Ding M, Gao X, Fan D, Zeng J, Wong K, Lu C, Xiao J and Yao C: Cilostazol as an alternative to aspirin after ischaemic stroke: a randomised, double-blind, pilot study. Lancet Neurol 7: 494-499, 2008

32. Shinohara Y, Katayama Y, Uchiyama S, Yamaguchi T, Handa S, Matsuoka K, Ohashi Y, Tanahashi N, Yamamoto H, Genka C, Kitagawa Y, Kusuoka H, Nishimaru K, Tsushima M, Koretsune Y, Sawada T and Hamada C: Cilostazol for prevention of secondary stroke (CSPS 2): an aspirin-controlled, doubleblind, randomised non-inferiority trial. Lancet Neurol 10: 959-968, 2010 